\title{
Metformin induced lactic acidosis - a case report
}

\author{
Onder N., Khishigsuren B.,Keskinorak N., Kutlu F., Seren S.
}

\section{Summary}

Metformin is a biguanid and because of its effects in decreasing the production of glucose in liver and in increasing the sensitivity of insulin in peripheral tissues like adipose tissue and skeletal muscle, it is used in metabolic syndrome and type 2 diabetes mellitus which insulin resistance is especially pronounced. The most important side effect of metformin is lactic acidosis. Lactic acidosis is the most frequent cause of hospitalized patients with metabolic acidosis. Especially renal dysfunction, liver disease, and in patients with risk factors such as heavy alcohol intake; It is recognized as a potential complication of metformin lactic acidosis. As a result, it can cause cardiopulmonary arrest. Systemic hypoxemia, sepsis, dehydration, old age and overdose cases increases in these complications.

A 60 year-old female patient applied to the emergency service because of the widespread burning body ache and general condition disorder. Results of examinations and laboratory; has been identified in patients with severe renal insufficiency and leukocytosis and invested with the suspecting of sepsis and was admitted to the intensive care unit. Mechanic ventilation and continuous venovenous hemofiltration was performed under deep sedation. Volume replacement, vasopressor and antibiotic therapy were also performed. Finally she was discharged to the service without any squelle.

Key Words: Metformin, Lactic acidosis, Obesity and Insulin Resistance

\section{Introduction}

Metformin has some advantage over the sulfonylurea group. For example; it does not cause hypoglycemia, does not increase hyperinsulinemia and weight gain and contributes positively to blood lipid levels. Because of these advantages, the metformin is widely used alone or in combination for the treatment of type 2 diabetes patients. The most important side effect of metformin is lactic acidosis. Though to abnormal renal function predisposing to lactic acidosis, it may also occur in individuals with normal renal function as well. Although the metformin induced lactic acidosis rare, since approximately $50 \%$ of mortality is a serious clinical situation. It should be considered in the patients, developing sudden respiratory failure admitted to the intensive care unit and especially use metformin

We aimed to discuss the effects of lactic acidosis due to inappropriate use of Metformin.

\section{Case presentation}

A 60 years old female patient was applied to the emergency service because of the widespread burning body ache and bad general condition. She was hypothermic and dehydrated with dry oral mucosa. Hemodynamic evidences were: blood pressure $160 / 70 \mathrm{mmHg}$, pulse 110/ min, respiratory rate $40 / \mathrm{min}$ and $\mathrm{SpO}_{2} 98 \%$. Medical history: controlled type 2 diabetes regulated with metformin $2 \mathrm{~g}$ daily for 5 years. The other medications were, losartan/ hydrochlorothiazide 100/12.5 daily for hypertension and $50 \mathrm{mcg}$ daily levothyroxine sodium for hypothyroidism. She had total abdominal hysterectomy and bilateral salpingooophorectomy 10 days ago and obesity surgery 7 months ago. She had no other system pathology evidence in our examination. Her initial investigations were as follows: BUN $69 \mathrm{mg} / \mathrm{dl}$ (normally 8 to $23 \mathrm{mg} / \mathrm{dl}$ ), and serum creatinine: $7.42 \mathrm{mg} / \mathrm{dl}$ (normally 0.50 to $0.90 \mathrm{mg} / \mathrm{dl}$ ). Her arterial blood gas $(\mathrm{ABG})$ measurements revealed a profound metabolic acidosis: $\mathrm{pH}: 6,77, \mathrm{PaCO}_{2}: 9,6 \mathrm{mmHg}, \mathrm{HCO}_{3}: 3.5 \mathrm{mmol} / \mathrm{L}$ and lactate: 18 $\mathrm{mmol} / \mathrm{L}$, ABE: -32.3 , glucose: $40 \mathrm{mg} / \mathrm{dl}$. Lung $\mathrm{x}$-ray and Electrocardiography (ECG) were normal.

She was sedated on the findings underwent and endotracheal intubation and mechanical ventilation was started. Her initial fluid resuscitation strategy included $2000 \mathrm{~mL} / 3 \mathrm{~h}$ of $0.9 \%$ balanced electrolyte solution and $1 \mathrm{~mL} / \mathrm{kg}$ of bolus $8.4 \%$ sodium bicarbonate solution was given. CVVHDF (continuous veno-venous hemodiafiltration) was started after nephrology consultation and nasal culture, rectal swabs, blood, urine, tracheal cultures were taken and Meronem1gx3 was began after infectious diseases consultation also general surgery consultation was performed, bowel sounds were evaluated normal and abdominal ultrasound scans were performed and acute pathology was not considered. As hypotension developed inotropic therapy was started. When her hemodynamic parameters recovered, were planned an abdominal CT. ABG were recovered rapidly, on the second day of hospital admission, $\mathrm{pH}$ : 7,36, $\mathrm{PaO}_{2}: 106, \mathrm{PaCO}_{2}: 33$, Lactate: $1.7, \mathrm{HCO}_{3}: 20$ creatinine: 1.95 . Patient was extubated and inotropic support reduced while hemodiafiltration was continued. Third day, after hemodynamic stabilization, inotropic treatment was stopped and fourth day hemodiafiltration was discontinued. Due to all cultures were negative, anti-microbial treatment was stopped. She was cooperated, oriented and has stable hemodynamics with adequate diuresis, she was started oral intake and discharged to the service.

\section{Discussion}

Metformin is widely used at metabolic syndrome and for treatment type 2 Diabetes Mellitus (DM). Lactic acidosis due to metformin is rare and preventable, but when it occurs it can be life threatening. Though it may occur especially in the patients with impaired renal functions should be also considered the patients without impaired renal functions as well.

The mechanism of lactic acidosis caused by metformin is unclear. Metformin decreases the pyruvate dehydrogenase activity and the mitochondrial transport of the consumed agents; therefore it increases the anaerobic metabolism. With the presence of decreased insulin release anaerobic metabolism increases, as a result the production of the Krebs cycles precursors increase. Inhibition of pyruvate dehydrogenase decreases the production of the precursors used for aerobic metabolism and causes transformation pyruvate to lactate. As a result of all these events the production of net lactic acid increases. In addition, increase of the glucose utilization in the small intestine due to metformin theoretically increases portal vein lactate levels and contributes to the development of lactic acidosis

Salpeter et all. retrospectively evaluated the metformin and non metformin oral antidiabetic trials which is made between 1959 and 2002, and they did not find any difference between two drug groups. Lactic acidosis divided into two groups; type A and B. First group is more frequent and, it may occur with or without hypoxia in patients with reduced tissue perfusion. Type B lactic acidosis may occur due to some drugs, chemicals, toxins, and lactate accumulation caused by genetic disorders. It is reported that one of the reasons of type B lactic acidosis is metformin. Clinically, severe type B lactic acidosis patients usually manifest shock and tissue hypoxia symptoms; therefore indiscrimination between type A and type B does not matter in diagnostic terms. Main factor of lactic acidosis in our case is not tissue hypoxia that exists before the use of metformin, but the tissue hypoxia contributes to lactic acidosis. Due to the developing lactic acidosis after the metformin use, we consider that the main factor of the lactic acidosis is metformin use in our case.

Lactic acidosis is an emergency situation that must be diagnosed and treated quickly. In the series of Misbin at all. \%42.5 mortality rate was reported. Recovery can be possible with early diagnosis and treatment in serious cases. It is reported that especially the patients with chronic renal failure have benefited from dialysis. Studies on this topic show that in terms of survival in patients with lactic acidosis due to metformin use, the most important treatments are high-volume hemodialysis and hemofiltration. Panzer et al. had reported one of the most interesting cases with lactic acidosis, in which they primarily started hemodialysis for the treatment of lactic acidosis. Our case also responded dramatically to hemofiltration.

\section{Conclussion}

As a result, lactic acidosis is described as a fatal complication of metformin with unclear mechanism. Mainstays of treatment are early diagnosis, correction of lactic acidosis, cardiovascular and respiratory support and normalization of the body temperature. The most important and effective treatment is the use of CVVHF. Because of the positive effect on insulin resistance, metformin can be used alone or combined with other drugs in type $2 \mathrm{DM}$ and have a potential of lactic acidosis side effect. This side effect is rare and preventable, but when occurs it can be mortal. For this reason, we believe that in elderly patients with severe diabetic complications, one should be careful when choosing metformin for treatment of type $2 \mathrm{DM}$.

References

Cesur M, Cekmen N, Cetinbas RR, Bedel P, Erdemli O. Lactic Acidosis Due to use of Metformin in a Patient with Diabetes Mellitus. Yogun Bakım Dergisi 2005;5(4):250-4

Fonseca V, Rosenstock J, Patwardhan R, et al. Effect of metformin and rosiglitazone combination therapy in patients with type 2 diabetes mellitus: A randomized controlled trial. JAMA 2000;283:1695-702. Gomez-Perez FJ, Fanghanel-Salmon G, et al. Efficacy and safety of rosiglitazone plus metformin in Mexicans with type 2 diabetes. Diabetes Metab Res Rev 2002;18:127-34. 6. Di Cicco RA, Allen A, Carr A, tal. Rosiglitazone does not alter the pharmacokinetics of metformin. J Clin Pharmacol 2000;40:1280-5.

Hulisz DT, Bonfiglio MF, Murray RD. Metformin-associated lactic acidosis. J Am Board Fam Pract 1998;11:233-6.

Hundal RS, Inzucchi SE. Metformin: New understandings, new uses. Drugs 2003;63:1879-94.

Salpeter S, Greyber E, Pasternak G, et al. Risk of fatal and nonfatal lactic acidosis with metformin use in type 2 diabetes mellitus. Cochrane Database Syst Rev 2002;(2):2967.

Khan JK, Pallaki M, Tolbert SR, et al. Lactic acidemia associated with metformin. Ann Pharmacother 2003;37:66-9.

Orban JC, Giunti C, Levraut J, et al. L'acidose lactique reste une complication grave du traitement par metformine metformin-associated lactic acidosis remains a serious complication of metformin therapy. Ann Fr Anesth Reanim 2003;22:461-5.

Wiernsperger NF, Bailey CJ. The antihyperglycaemic effect of metformin: Therapeutic and cellular mechanisms. Drugs 1999;58:31-9. 